\title{
L'impact des carences affectives vues par les neurosciences
}

\begin{abstract}
:
In the early twentieth century, the work of Spitz psychoanalysts Bion, Anna Freud and Winnicott drew attention to the psychological problems related to emotional deprivation in identity construction. John Bowlby (1969), author of the theory of attachment, considers the trend to establish close relationships as essential to the survival of the human being. Today, with advances in MRI material, it is possible to understand how the mechanisms of the lack of attachment, in other words, emotional deprivation, and shall adjust to impact our brain. These new neurophysiological data confirm the importance of maternal care on child development, especially during the first years of life.
\end{abstract}

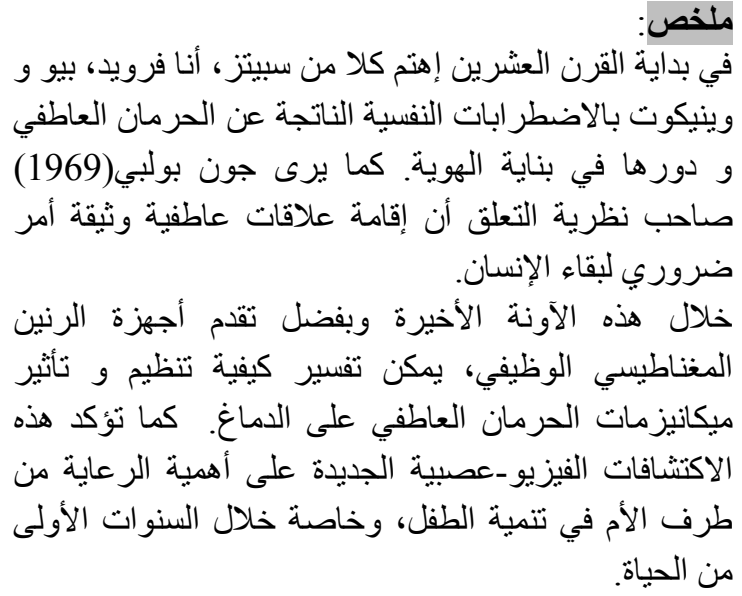

\author{
Dr. Nafissa Ghanem \\ Département de psychologie \\ Université Abdel Mehri \\ Constantine 2
}

\section{Introduction :}

Au cours des dernières années, plusieurs recherches tendent à confirmer que le besoin primordial du jeune enfant et spécialement du nourrisson est d'établir un lien permanent et sécurisant avec une figure maternelle ou un substitut répondant à ses besoins.

Selon Kreisler (1987), afin de réaliser un climat affectif harmonieux, le nourrisson doit pouvoir bénéficier, à travers les échanges avec sa mère ou son substitut : 
-une plénitude des apports affectifs,

-une souplesse d'adaptation à ses besoins,

- une stabilité de la relation.

Pour désigner ce lien particulier qui unit l'enfant à la figure maternelle, John Bowlby (1907 - 1990), psychanalyste, propose le terme «d'attachement ».

Ayant été influencé par la théorie éthologique, mais surtout par l'étude de l'empreinte de Lonrenz (1935; cité par Bowlby, 1989) qui a démontré que l'attachement était inné(chez les jeunes canetons) et a donc une valeur de survie, Bowlby a élaboré sa "théorie de l'attachement » qui suggère que les enfants viennent au monde biologiquement pré- programmé pour former des pièces jointes avec les autres, parce que cela va les aider à survivre. Il a égalementestimé que la santé mentale et les problèmes comportementaux peuvent être attribués à la petite enfance.

\section{Les fondements de la théorie de l'attachement :}

Le psychiatre et psychanalyste anglais John Bowlby (1969) a travaillé,avant la guerre, sur les séparations, la perte et le deuil.

Il a ensuite mit en évidence les mécanismes de formation et de développement des relations d'attachement en intégrant, en 1969, les apports de la psychanalyse et ceux de l'éthologie dans sa théorisation des liens d'attachement.

En effet, la seconde guerre mondiale amène les psychiatres et les éthologues de cette période à s'intéresser au lien mère-enfant et aux conséquences des séparations précoces.

Les principaux travaux à l'origine de cette théorie sont ceux de l'éminent médecin et psychanalyste viennois Spitz (1947), et des éthologues Harlow (1958) et Lorenz (1970). Les études de Spitz, connues sous le nom d'" hospitalisme», qui seront citées plus en détail ultérieurement, démontrent l'impact de la relation mère-enfant sur leur séparation.

Spitz y parle pour la première fois de symptômes dépressifs du nourrisson et montre que plus la relation mère-enfant a été chaleureuse et aimante, plus la rupture sera dramatique. Celles de Harlow (1958), réalisées sur des singes macaques, séparés de leur mère, vont révéler que la recherche de contacts (et donc du réconfort que ceux-ci impliquent) est plus importante que la recherche de nourriture. Enfin, celles de Lorenz (1970), connues sous le nom de «phénomène de l'empreinte», confirment la fonction adaptative du lien d'attachement qui se crée entre le jeune animal et sa mère ou son substitut.

Bowlby (1969) décrit l'attachement comme étant le produit des comportements qui ont pour objet la recherche et le maintien de la proximité d'une personne spécifique. 
C'est un besoin social primaire et inné d'entrer en relation avec autrui. En ce sens, il s'éloigne de Freud pour lequel les seuls besoins primaires sont ceux du corps, l'attachement de l'enfant n'étant qu'une pulsion secondaire qui s'étaye sur le besoin primaire de nourriture.

Selon Bowlby, la mère, ou son substitut, constitue une base de sécurité pour son enfant. Le nouveau-né dispose d'un répertoire de comportements instinctifs, tels que s'accrocher, sucer, pleurer, sourire, qui vont pouvoir être utilisés au profit de l'attachement. Car le facteur déterminant de l'attachement n'est pas la nourriture, mais les soins et la réactivité

La fonction de l'attachement est pour Bowlby (1969) une fonction adaptative à la fois de protection et d'exploration. La figure d'attachement va agir comme une base sûre pour explorer le monde,de même qu'elle servira de prototype pour toutes les futures relations sociales et qu'une quelconque perturbation peut avoir de lourdes conséquences.

\section{Les carences affectives :}

En effet, tous les nouveaux nés ne bénéficient pas ou bénéficient peu d'une figure d'attachement, ce qui aura de lourdes conséquences sur le reste de leur vie.Dans cette optique, nous rapportons ici les toutes premières études ayant démontré l'impact de la privation affective, sans pour autant passer en revue le nombre croissant d'études qui impacteun large choix de troubles comportementaux auxcarencesaffectives :

La première étude est celle de Goldfarb(1943) qui a ciblé deux groupes d'enfants. Le premier groupe, selon l'auteur, était socialement isolé. Il s'agissait de 15 enfants âgés de 6 mois à 3 ans élevés dans une institution pour enfants abandonnés. Le deuxième groupe, également constitué de 15 enfants du même âge, élevéesen familles d'accueil. En prenant en considération le quotion intellectuel, la pensée abstraire et la maturité sociale, l'étude a conclu que legroupe «institution» était à la traîne en comparaison au groupe « famille d'accueil ».

- Quelques années plus tard, Spitz \& Wolf (1946) étudièrent les enfants placés dans les orphelinats et les hôpitaux. Spitz rapporta que les institutions étaient de mauvaise qualité et que le personnel interagissait rarement avec les enfants. Le constatétait qu'un tiers des enfants placés en institution étaient morts avant l'âge de 1 an. Quant au reste des enfants, ils avaient montré des signes de «dépression anaclitique »: de l'apathie, un retrait social, et unretard psychomoteur. L'étude à également montré que ces symptômes s'estompaient si la période de privation maternelle était inférieure à trois mois.

Spitz a également comparé les enfants vivant dans un orphelinat avec d'autres vivants dans un établissement pénitentiaire où 
ils ont été pris en charge par leurs mères. Bien que les conditions physiques dans l'orphelinat étaient meilleures, les enfants avaient un développement inférieur. Deux ans plus tard, 37\% des enfants de l'orphelinat étaient morts alors que 5 ans plus tard, tous les enfants élevés par leurs mères dans la prison étaient encore en vie.

De nos jours les neuro sciences ont bouleversé le monde de la psychologie. En effet, un certain Rizzollati a mis le doigt sur une découverte révolutionnaire qui constituerait un élément central dans les carences affectives il s'agit des «neurones miroirs».

\section{Découvertes des neurones miroirs :}

Un jour en 1990, Giacomo Rizzolatti, directeur du département de neurosciences de la faculté de médecine de Parmeet son équipe de chercheurs travaillaient avec des singes portant des casques à résonnance magnétique. Lors de la pause déjeuner, celui-ci tendit la main droite vers un sandwich: le cerveau du singe qui le regarde fait crépiter le casque. Le chercheur arrête son geste, puis le recommence ; à nouveau crépitation. L'IRM lui montre que le singe, resté immobile, envoie de l'énergie à son cerveau comme si c'était lui qui levait la main droite pour attraper le sandwich. Il venait de découvrir le principe des neurones miroirs.

C'est ainsi, par hasard, que Rizzolatti découvre que des neurones placés dans la zone F5 du cortex moteur, activés quand le macaque exécute une action, sont également activés chez un autre macaque qui observe son congénère, sans pour autant effectuer l'action. Ce constat a fait dire à Rizzolatti que ces neurones servent à projeter une représentation de l'action, que celle-ci ait lieu ou non. Autrement dit, pour comprendre ce que fait l'autre, un sujet doit activer ses propres neurones moteurs qui en situation d'agir, seraient activés pour réaliser la même action que celle déployée par l'individu observé.

«Les neurones miroirs constituent une classe particulière de neurones initialement identifiés dans le cortex précentral du macaque. Leur caractéristique principale est de s'activer aussi bien lorsque le singe effectue une action spécifique ou lorsqu'il observe un autre individu en train d'exécuter la même action. Ainsi un tel neurone s'active quand le singe saisit un objet donné, ou lorsqu'il voit l'expérimentateur saisir le même objet. Certains de ces neurones sont très spécifiques, ne s'activant que si les deux mouvements, saisie observée et saisie exécutée, sont réalisés de la même façon (...) Quel est le rôle fonctionnel des neurones miroirs? Diverses hypothèses ont été avancées. En fait, leur fonction n'est pas unique. Leur propriété est de constituer un mécanisme qui projette une description de l'action, élaborée dans les aires visuelles complexes, vers les zones motrices. Ce mécanisme de transfert comporte toute une variété d'opérations. Une de leurs fonctions essentielles est la compréhension de l'action. Il peut paraître bizarre que, pour reconnaître ce 
que l'autre est en train de faire, on doive activer son propre système moteur. En fait, cela n'est pas tellement surprenant. Car la seule observation visuelle, sans implication du système moteur, ne donne qu'une description des aspects visibles du mouvement, sans informer sur ce que signifie réellement cette action. Cette information ne peut être obtenue que si l'action observée est transcrite dans le système moteur de l'observateur. L'activation du circuit miroir est ainsi essentielle pour donner à l'observateur une compréhension réelle et expérientielle de l'action qu'il voit » (Rizzolatti, conférence donnée à l'Académie des sciences, déc. 2006)

Depuis 1996, la présence des neurones miroirs chez l'homme a été confirmée par nombres d'études. De même qu'il a été observé la présence des neurones miroirs dans le cerveau encore immature du jeune enfant. Et chez l'adulte, ces réseaux miroirs apparaissent comme bien plus développés que chez les autres primates. Ce détail semble anodin et couler de source puisque le cerveau de l'homme est bien plus gros que celui des singes. Mais le fait que les neurones miroirs y soient très développés n'est pas fortuit. Car tout dispositif naturel possède une contrepartie fonctionnelle et si ces neurones sont présents en nombre, c'est sans doute parce qu'ils ont un lien avec ce qui sépare l'homme de l'animal «la raison et le langage». Et plus généralement, l'intersubjectivité.

La découverte des neurones miroirs a fait émerger un nouveau concept "l'intelligence relationnelle» ou ce qu'on appelle également" le cerveau neurosocial». en effet, Un certain nombre de chercheurs (comme les psychologues Frans de Waal, Jean Decetyet Vittorio Gallese) pensent que les neurones miroirs jouent un rôle important dans l'empathie, c'est-à-dire dans la capacité à percevoir et reconnaître les émotions d'autrui, le système miroir des émotions permet de simuler l'état émotionnel d'autrui dans notre cerveau et donc de mieux identifier les émotions éprouvées par les individus de notre entourage.Nous reprenons ici Cyrulink (2006) qui rapporte que: «nos neurones entrent sans arrêt en résonnance avec ceux d'autrui ; nos intériorités sont en communication directe. Nos circuits neuronaux sont fait pour se mettre en phase avec ceux des autres ». En effet, si jadis, Freud avait parlé d'inconscient immatériel, récemment, et grâce aux progrès de l'imagerie fonctionnelle cérébrale, de la neuro-imagerie, on a découvert un inconscient biologique, qui nous révèle que sans la présence d'un autre, nous ne pouvons pas devenir nous-même : «Sans attachement, pas d'empathie. Le " je " ne peut pas vivre seul ». (Cyrulink,, 2006)

En d'autres termes, un cerveau seul, même sain, ne fonctionnerait pas, il lui faudrait au moins un autre cerveau pour se développer.Dès le plus jeune âge, le cerveau de l'enfant aura besoin d'informations sensorielles pour le structurer. L'enfant aura besoin d'interactions sociales, de percevoir, d'identifier les 
émotions des autres pour ensuite les imiter. Mais qu'en est-il des enfants dont le milieu sensoriel est appauvrit?

Hélas, tous les enfants n'ont pas la chance de naitre dans des familles unies, ou avec des parents démonstratifs qui consacrent une partie de leur temps àborder leurs progénitures, ce qui va, ultérieurement, porter préjudice au développement cérébral de ces derniers.

Durant les deux premières semaines de son existence, quand la mère du nouveau née est défaillante (pour cause de décès ou de maladie), et quand le nouveau-né n'est pas correctementpris en charge par un substitut maternel, le milieu sensoriel de l'enfant est alors appauvri. Ce manque prive l'enfant des stimulations biologiques initiales, ce qui atrophie son cerveau puisque ses neurones sont mois sollicités.

En effet, l'imagerie médicale a démontré qu'à la naissance, le système nerveux, et particulièrement le cerveau, de l'être humain est nettement moins développé que chez les autres primates. Le poids du cerveau du nouveau- né se multipliera par 4 durant la première année de sa vie.

Aussi, il a été prouvé que, chez le nouveau -né, la plupart des neurones ne sont pas encore reliés les uns aux autres et que c'est en réponse aux stimulations provenant de son environnement que vont se créer des contacts dendritiques entre les cellules nerveuses et que les neurones se connecteront. Cette connexion entre deux neurones s'appelle une synapse.

Durant la première année de vie, des synapses continueront à évoluer, de nouveaux réseaux de neurones seront créés. A chaque fois que l'enfant fera de nouvelles découvertes, des connexions se formeront, d'autres se renforceront, d'autres s'affaibliront et certaines disparaitront. L'efficacité des synapses est donc influencée par les informations qui sont reçues par le cerveau ( Dubois J, et al., 2011) .

Il est clair que les soins et l'attention donnés à un enfant sont primordiaux. Ils assurent non seulement son bien-être et sa survie, mais sont également essentielles au développement de son cerveau. Si l'enfant est dans un milieu stimulant, ses apprentissages et son développement seront alors beaucoup plus importants. A ce sujet Cyrulink (2006) affirme que c'est sous l'effet des interactions précoces que le cerveau acquiert une manière d'être sensible au monde et d'y réagir. Il ajoute que les neurones de l'hippocampe sont les plus réactifs à ce processus qui joue un rôle important dans les circuits de la mémoire et dans l'acquisition des aptitudes émotionnelles.

Ces données neurologiques permettent de comprendre en partie pourquoi une carence affective précoce, qui atrophie le cerveau et contrarie la connexion de neurones tels que les neurones miroirs, qui permettent de mimer les émotions d'autrui, entraînerait un mauvais traitement des informations, un mauvais contrôle des émotions, et par conséquent un comportement mal adapté. 
Par ailleurs l'autre cause des atrophies cérébrales provient des molécules du stress appelées «Cortisol» que sécrète l'enfant sous l'effet des conditions environnementales et qui font éclater le corps cellulaire des neurones.

De récentes études en neurophysiologie ont démontré que les enfants dont on s'est occupé de façon moins constante, moins chaleureuse et moins affectueuse produisent plus de cortisol.

La plus diserte de ces études est celle effectuée par le Dr Gunnar (Université de Minnesota) sur 151 enfantsâgés de 0 à 5 ans, suite à laquelle il a été observé une augmentation decortisol salivaire chez la majorité des enfants $(63 \%)$ par rapport aux niveauxde cortisoldes enfants gardés à la maison. Cette même hormone inhibe la croissance et serait responsable de troubles anxieux mais aussi de maladies auto-immunes et inflammatoires, de dépression ou de toutes formes de dépendances.

Le Dr. Perry et ses collègues du Baylor Collègeà Houston(1995)démontrent pour leur part que les nourrissons et les jeunes enfants maltraités et négligés sont plus susceptibles de produire une forte réaction au stress. Ces jeunes enfants présentent, également,une atrophie de l'amygdale qui joue un rôle essentiel dans la gestion des émotions, car c'est elle qui nous fait réagir en une fraction de seconde à la suite d'un stimulus menaçant et qui active notre mémoire émotionnelle.

\section{Conclusion}

Enfin, ces notions récentes de neuroscience démontrent que le modelage du cerveau et la connexion synaptique sont la conséquence de la rencontre entre un point de départ génétique qui donne le cerveau et d'un apport sensoriel organisé par les comportements parentaux.

Heureusement, rien n'est figé dans le développement et le devenir de l'enfant. Actuellement, nous assistons à l'émergence d'un nouveau concept qui vient ébranler tout prérequis. Les progrès des neurosciences ont mis le doigt sur ce qu'on appelle « la plasticité neuronale».

Nous savons aujourd'hui que le cerveau humain est un système malléable, en perpétuelle reconfiguration, qui a la capacité de se modifier et de se reconfigurer sous l'influence favorable du milieu sensoriel, affectif, social et culturel dans lequel nous évoluons tout au long de notre vie, et ce, quel que soit notre âge.

\section{Biblioghraphie:}

1- Bowlby,j. (1969). Attachement et perte. tome 1, Puf.

2-Bruce, et al (1995). Childhood trauma, the neurobiology of adaptation, and un dependent" development of the brain: how "statets" becom"traits", in infant health mental journal, Vol. 16, No. 4.

3-Cyrulink, B. (2006).De chair et d'âme. Edition Odile jacob. 
4-Dubois, J et al. (2011) Neurophysiologie clinique : développement cérébral $\mathrm{du}$ nourrisson et imagerie par résonance magnétique.In Neurophysiologie Clinique.

5- Gunnar, R et al. (2010). The Rise in Cortisol in Family Daycare: Associations With Aspects of Care Quality, Child Behavior, and Child Sex.In US National library of medicine. En ligne: http://www.ncbi.nlm.nih.gov/pmc/articles/PMC2946618/

6 - Harlow, j. (1958). The Nature of Love. in American Psychologist, 13, 673685.

7- Kreisler, (1987), Le nouvel enfant du désordre psychosomatique. Toulouse. Privat.

8- Rizzolatti, G. (2009). conférence donnée à l'Académie des sciences, déc. 2006).

En ligne : https://www.neurosciences.asso.fr/V2/Actualites/LaLettre/Let32.pdf 9- Spitz R.A.. (1945). Hospitalism : An inquiry into the genesis of psychiatric conditions in

early childhood. The Psychoanalytic Study of the Child. 145 ; 1:53-74 